CASE REPORT

\title{
Fulminant Crohn's colitis after allogeneic stem cell transplantation
}

\author{
S A Sonwalkar, R M James, T Ahmad, L Zhang, C S Verbeke, D L Barnard, D P Jewell, M A Hull
}

Gut 2003;52:1518-1521

We report a case of fulminant Crohn's colitis that occurred following non-myeloablative allogeneic stem cell transplantation for Hodgkin's lymphoma. Adoptive transfer of inflammatory bowel disease by haematopoietic cells is recognised in several animal models of inflammatory bowel disease and remission of Crohn's disease has been reported in patients who have received a bone marrow transplant. However, adoptive transfer of Crohn's disease susceptibility leading to phenotypic manifestation of the disease after transplantation has not been previously reported. Having ruled out an infective cause of a colitis in this case, we speculated that adoptive transfer of Crohn's disease may have occurred and performed a genetic analysis of known susceptibility loci for significant donor-recipient mismatches. The donor and recipient had several haplotype mismatches in HLA class III genes at the IBD3 locus. In addition, the donor (but not the recipient) had a polymorphism of the $5^{\prime}$ UTR of NOD2/CARD15 that may be associated with Crohn's disease. This case highlights the question of whether adoptive transfer of Crohn's disease can occur between allogeneic stem cell transplant donor and recipient, in a similar fashion to that reported for other autoimmune diseases. This report should also stimulate debate regarding the need for stem cell transplant donor screening for inflammatory bowel disease.

urrent dogma suggests that the phenotypic manifestations of Crohn's disease (CD) arise when an inappropriate intestinal immune response occurs to an environmental stimulus or stimuli in a genetically susceptible individual. ${ }^{1}$ The recent description of several genetic susceptibility loci for $\mathrm{CD}$ has emphasised the significant role of the host genotype in determining individual CD risk. ${ }^{2}$ In keeping with the pivotal role of intestinal immune dysregulation in the aetiology of $C D$, the first characterised CD susceptibility gene NOD2/CARD15 is expressed in monocytes, in which it may act as an intracellular lipopolysaccharide receptor. ${ }^{2}$ If a significant contribution to CD pathogenesis is explained by polymorphism(s) in genes that are expressed predominantly in cells of haematopoietic origin, allogeneic stem cell transplantation (ASCT) could allow transfer of CD susceptibility between individuals. Several cases of long term remission of CD in ASCT recipients have been described. ${ }^{34}$ However, adoptive transfer of CD susceptibility, leading to the phenotypic manifestation of CD in an ASCT recipient, has not been described previously. Herein, we report a case of fulminant Crohn's colitis that occurred following ASCT. Development of CD after ASCT has important implications for our understanding of $\mathrm{CD}$ pathogenesis, as well as for screening of potential ASCT donors.

\section{CASE REPORT}

A 34 year old female first presented in 1993 with a history of tiredness, shortness of breath, night sweats, and rapid weight loss. Computed tomography (CT) of the thorax revealed an anterior mediastinal mass, biopsy of which demonstrated the typical features of nodular sclerosing Hodgkin's lymphoma stage IIIB.

A standard chemotherapy regimen was initiated, resulting in a partial response. Her subsequent clinical course, over the next seven years, was characterised by temporary benefit from radiotherapy and many chemotherapy regimens, including high dose therapy with autologous rescue, all followed by disease relapse. Subsequently, she underwent non-myeloablative ASCT in June 2001. A conditioning regimen of Campath-1H, fludarabine, and melphalan was used. The donor and recipient were matched exactly for HLA class I (A2 A3 B61[B40] B62[B15] Bw6 Cw2 Cw10) and HLA class II (DR4 DR14[DR6] DQ5 DQ8 DRw52 DRw53) alleles. Testing for human immunodeficiency virus confirmed that both donor and recipient were negative. Cyclosporin therapy was commenced on the day of the transplant. Engraftment was successful, and chimerism studies at six months post ASCT confirmed the presence of $100 \%$ donor lymphocytes in recipient peripheral blood. CT scan of the thorax six months after ASCT demonstrated complete resolution of the mediastinal mass.

Three months post ASCT, she developed stage I cutaneous graft versus host disease (GvHD) which responded to a short course of corticosteroid therapy. Two weeks later she developed colicky abdominal pain and bloody diarrhoea. Routine stool culture for enteric pathogens, including Clostridium difficile, was negative. Her symptoms settled spontaneously within one week. Six months after ASCT, she developed further abdominal pain and bloody diarrhoea, which necessitated hospitalisation. The patient denied any history of abdominal pain or diarrhoea, prior to ASCT. There was no history of previous oral ulceration, skin rashes, or ophthalmological disease. There was no family history of inflammatory bowel disease (IBD). The patient was a nonsmoker. She was taking cyclosporin $50 \mathrm{mg}$ twice daily, acyclovir $200 \mathrm{mg}$ twice daily, fluconazole $50 \mathrm{mg}$ once daily, co-trimoxazole $400 \mathrm{mg}$ once daily, omeprazole $20 \mathrm{mg}$ once daily, and combined oestradiol $1 \mathrm{mg}$ /norethisterone $1 \mathrm{mg}$ once daily. There was no history of non-steroidal antiinflammatory drug use. On examination, she had a low grade pyrexia (temperature $37.4^{\circ} \mathrm{C}$ ), patchy dry red skin (prominent on the neck and elbows), diffuse mild abdominal tenderness, and marked aphthous oral ulceration. Investigations revealed a peripheral blood total white cell count of $12.1 \times 10^{9} / 1$ (normal range $4.0-11.0 \times 10^{9} / 1$ (neutrophils $\left.6.5 \times 10^{9} / 1\right)$ ), thrombocytosis (platelet count $469 \times 10^{9} / 1$; normal range

Abbreviations: CD, Crohn's disease; ASCT, allogeneic stem cell transplantation; $\mathrm{CT}$, computed tomography; GvHD, graft versus host disease; IBD, inflammatory bowel disease; CRP, C reactive protein; CMV, cytomegalovirus; HSV, herpes simplex virus; SNP, single nucleotide polymorphism 
140-380 $\times 10^{9} / 1$ ), raised $C$ reactive protein (CRP) level of $220 \mathrm{mg} / \mathrm{l}$ (normal range $<10 \mathrm{mg} / \mathrm{l}$ ), and decreased serum albumin level of $29 \mathrm{~g} / \mathrm{l}$ (normal range 35-65 g/l). Cyclosporin levels were within the therapeutic range. Stool microscopy and culture for Clostridium difficile (and toxin), Campylobacter jejuni, Yersinia enterocolitica, Shigella spp, Salmonella spp, cytomegalovirus (CMV), Epstein-Barr virus, rotavirus, Norwalk virus, Cryptosporidium, and amoebae were negative. A supine plain abdominal $x$ ray revealed a normal bowel gas pattern. A skin biopsy from the elbow was not diagnostic of GvHD but demonstrated features of a non-specific dermatitis.

Flexible sigmoidoscopy revealed purulent discharge, contact bleeding, and inflamed mucosa with loss of the normal vascular pattern and multiple serpiginous ulcers, up to the point of insertion at the splenic flexure. Multiple colorectal biopsies demonstrated patchy transmucosal inflammation with deep ulceration and mucin depletion, as well as crypt distortion and crypt abscess formation (fig 1A). Several small non-caseating epithelioid granulomata were also evident. Crypt epithelial apoptosis, crypt destruction, and intraepithelial lymphocytosis were not present. No viral inclusion bodies or giant cells were seen. A diagnosis of Crohn's colitis was made. She was treated with intravenous hydrocortisone $100 \mathrm{mg}$ four times daily for three days, which was subsequently changed to oral prednisolone when she was discharged home (see below). Treatment with mesalazine $800 \mathrm{mg}$ orally three times daily was also initiated together
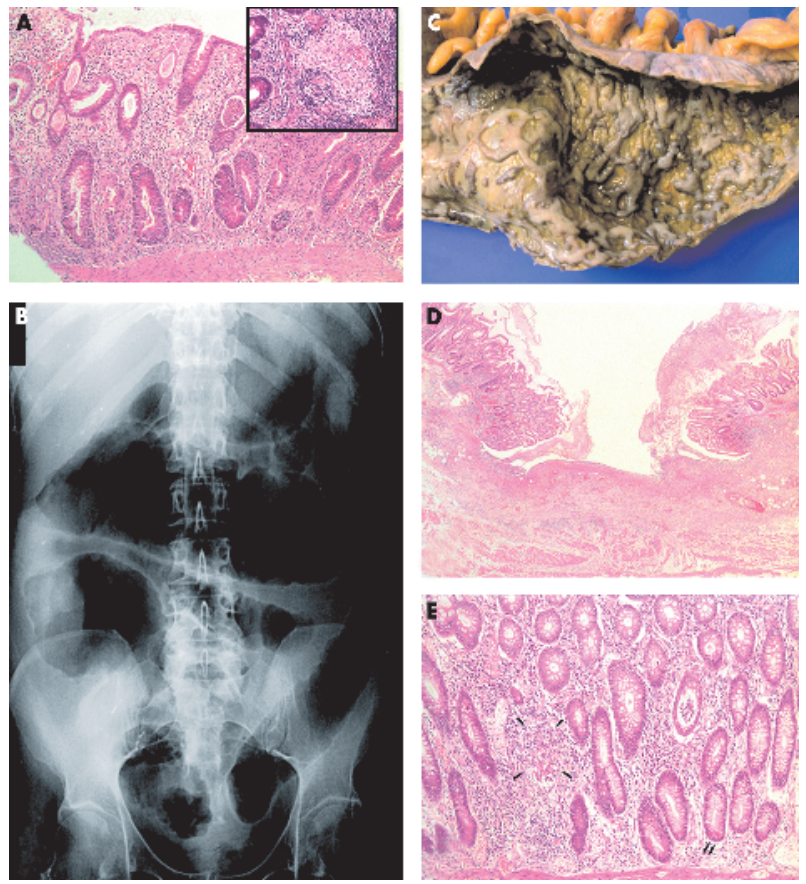

Figure 1 (A) Colonic biopsy showing crypt distortion, patchy transmucosal inflammation, crypt abscesses, and mucin depletion. Inset shows an epithelioid granuloma in the deep mucosa (haematoxylin and eosin; magnification $\times 100$ and $\times 200$ (inset)). (B) Supine plain abdominal $x$ ray with features of toxic megacolon. (C) Macroscopic appearance of the colectomy specimen revealing confluent geographical ulcers covered with pus and separated by small irregular islands of residual mucosa. (D) Microscopy of the colectomy specimen confirming the presence of broad ulceration flanked by inflamed mucosa displaying crypt distortion (haematoxylin and eosin; magnification $\times 25$ ).

(E) Colonic mucosa containing irregular and bifid crypts, occasional epithelioid granulomata (arrows), metaplastic Paneth cells (double arrow), and crypt abscesses (haematoxylin and eosin; magnification $\times 100$ ). with rectal prednisolone and mesalazine $(250 \mathrm{mg})$ twice daily. Cyclosporin was continued.

Abdominal pain and bloody diarrhoea improved dramatically such that, at the patient's request, she was discharged home three days later, with review planned in the outpatient clinic in seven days. However, she was readmitted after only 48 hours with profuse bloody diarrhoea and severe abdominal pain. On examination she had a tachycardia (pulse rate 130 beats/min), she was hypotensive (blood pressure 75/ $45 \mathrm{~mm} \mathrm{Hg}$ ), pyrexial (temperature $38.4^{\circ} \mathrm{C}$ ), and dehydrated. Her abdomen was tender with guarding and rigidity. Bowel sounds were absent. Blood investigations revealed a raised total white cell count of $13.2 \times 10^{9} / 1$ (neutrophils $8.7 \times 10^{9} / 1$ ), and a raised CRP level of $192 \mathrm{mg} / \mathrm{l}$. A supine plain abdominal $x$ ray showed marked dilatation and wall thickening of the colon, indicative of a toxic megacolon (fig 1B). An abdominal CT scan excluded any focus of extracolonic sepsis. Stool culture for enteric pathogens was again negative. She did not respond to 48 hours of intensive medical treatment with intravenous hydrocortisone $100 \mathrm{mg}$ four times daily, intravenous ciprofloxacin $750 \mathrm{mg}$ twice daily, intravenous metronidazole $500 \mathrm{mg}$ three times daily, and fluid resuscitation.

Her abdomen remained distended and tender with no resolution of the radiological findings on the plain supine abdominal $x$ ray. Therefore, she underwent an emergency subtotal colectomy with end ileostomy formation. At laparotomy, the small bowel was macroscopically normal. On gross examination, the entire resected colon was markedly dilated and the mucosal surface contained multiple confluent geographical ulcers that were covered with pus and that were separated by small irregular islands of residual mucosa (fig lC). Histological examination demonstrated numerous "flask"-shaped and a few "knife"-like ulcers, which extended into the submucosa and which were covered with abundant fibrinoneutrophilic exudate (fig lD). In areas of mucosal preservation, the colonic mucosa showed patchy changes including crypt irregularity, Paneth cell metaplasia, mild mucin depletion, chronic inflammatory cell infiltration, and crypt abscess formation. Occasional epithelioid granulomata were present within the deep mucosa (fig 1E). There was no evidence of fibrosis, vasculitis, or acute GvHD. No viral inclusions were seen. Immunohistochemistry for CMV and herpes simplex virus (HSV) type 1 and 2 was negative. Subsequent polymerase chain reaction analysis for the presence of HSV type 1 and 2 DNA, in three separate blocks of the colonic resection specimen, was also negative. ZiehlNeelsen staining revealed no acid fast bacteria in the colonic resection specimen.

Her postoperative course was uncomplicated and she made an uneventful recovery except for continuing oral HSV type 1 infection that eventually required oral and then intravenous antiviral therapy.

\section{DISCUSSION}

We believe that the ASCT recipient developed fulminant CD of the colon. ${ }^{5}$ This diagnosis was based on characteristic macroscopic and histological findings of Crohn's colitis, coupled with a lack of evidence for an alternative cause of fulminant colitis. Histological features were not compatible with acute or chronic colonic GvHD, which are characterised by crypt epithelial cell apoptosis and segmental crypt loss, or fibrosis of the submucosa, with or without a dense lymphocytic infiltrate, respectively. ${ }^{67}$ We were careful to exclude an infective colitis in an immunosuppressed ASCT recipient receiving cyclosporin. In particular, HSV colitis is recognised to occur in ASCT recipients and the histological features of HSV colitis can mimic those of CD. ${ }^{8}$ Therefore, we excluded this diagnosis by performing polymerase chain 
reaction analysis for HSV DNA and immunohistochemistry for HSV protein on formalin fixed sections of the colonic resection specimen. Finally, the patient was not neutropenic and the pathological findings were not consistent with neutropenic enterocolitis. ${ }^{10}$

The ASCT recipient reported no past history or family history of IBD, suggesting the intriguing possibility of a causal relationship between ASCT and development of CD several months later. It is possible that changes in an environmental factor, such as intestinal bacterial content, could have triggered the onset of CD in a susceptible host following ASCT. However, the absence of overt CD in the recipient during previous chemotherapy and antibiotic courses before ASCT would perhaps mitigate against a simple environmental trigger, in this case. Alternatively, limited clinical observations and data from animal models of IBD suggest that adoptive transfer of CD susceptibility by ASCT could also explain development of CD de novo.

Adoptive transfer of autoimmune thyroid disease, vitiligo, and psoriasis from ASCT donor to recipient has previously been reported. ${ }^{11-13}$ In addition, two cases have been reported in which adoptive transfer of ulcerative colitis may have occurred. ${ }^{14}{ }^{15}$ Histology characteristics of ulcerative colitis have been described in a 27 year old male who developed bloody diarrhoea 35 months after ASCT from his HLA matched brother.$^{14}$ However, this patient was later diagnosed with acquired immunodeficiency syndrome, thereby casting doubt on the original diagnosis. ${ }^{16}$ Ulcerative colitis has also been described in a 37 year old male seven months after ASCT from his HLA matched sibling, who had severe CD. ${ }^{15}$ The opposite outcome after ASCT (that is, cure of existing immune mediated disease) is perhaps more widely recognised. ${ }^{17}$ The literature includes a series of five patients with active CD who all received ASCT for haematological disease. ${ }^{3}$ Four of these individuals entered into long term CD remission. ${ }^{3}$ In keeping with a causal relationship between ASCT and induction of CD remission (rather than, for example, an association with a change in immunosuppressive therapy), the one individual who suffered a relapse of CD had significant haematopoietic chimerism post ASCT. ${ }^{3}$ Several rodent models also provide evidence that adoptive transfer of bone marrow derived cells can recapitulate an IBD-like enteritis in a wild-type recipient. These include the human HLA-B27/ $\beta_{2}$ microglobulin transgenic rat $^{18}$ and the interleukin 2 knockout mouse. ${ }^{19}$ Similarly, transfer of naïve $\mathrm{CD}_{4} \mathrm{Rb}^{\text {high }}$ lymphocytes into SCID recipient mice leads to development of gastrointestinal inflammation. ${ }^{20}$

Seeking further evidence that adoptive transfer may have occurred, we obtained the prior approval of the St James's and Seacroft University Hospitals Research Ethics Committee and approached the ASCT donor (while maintaining complete donor anonymity) and recipient in order to gain permission to perform further analysis of their stored peripheral blood DNA and obtain an accurate family history using the Oxford IBD questionnaire. ${ }^{2}$ Peripheral blood DNA from the donor and recipient (pre and post ASCT) was subsequently available for analysis.

We then performed detailed genetic analysis of three CD susceptibility loci (IBD1, chromosome 16; IBD3, chromosome 6; IBD5, chromosome 5) in donor and recipient samples. Genotyping of markers at all three loci confirmed complete donor haematopoietic reconstitution of the recipient without evidence of any chimerism. We screened donor as well as pre ASCT recipient DNA samples for polymorphisms in the 11 exons and intron-exon junctions of NOD2/CARD15 by direct sequencing, as described previously. ${ }^{21}$ Neither the donor nor the recipient (pre ASCT) possessed any of the three common disease associated NOD2/CARD15 variants (R702W, G908R, 1007fsinsC) or any of the 27 more rare NOD2/CARD15 mutations that have been described. ${ }^{21}$ This is perhaps not surprising as NOD2/CARD15 mutations seem to be associated with ileal and stenosing CD. ${ }^{22}$ However, the donor and recipient were mismatched for a $5^{\prime}$ UTR polymorphism that has been shown, in a single study, to be possibly associated with CD (it has not been excluded that this weak association is due to linkage disequilibrium with the three common $C D$ associated NOD2/CARD15 polymorphisms). ${ }^{21}$ At position -33, the donor (and post ASCT recipient DNA) was homozygous for the disease associated $\mathrm{T}$ allele while the pre ASCT recipient was homozygous for the wild-type $G$ allele. In contrast with NOD2/CARD15, HLA haplotypes associated with CD susceptibility at IBD3 (chromosome 6) confer a modest overall risk and may be most important in colonic disease. ${ }^{2}$ Haplotype diversity and extensive polymorphism within the gene dense HLA complex on chromosome 6 means that routine molecular matching of classical HLA class I and II genes between donor and recipient does not necessarily exclude mismatches of non-classical HLA class III gene haplotypes. High resolution HLA class I and II molecular typing for $H L A-A, B, C W, D R B 1, D R B 3, D R B 4, D R B 5$, and $D P B 1^{23}$ confirmed that donor and pre ASCT recipient DNAs were matched, with the exception of $H L A-D P B I$ and $H L A-B$, where a novel allelic variant was identified in the recipient. The DNA samples were further genotyped for 320 single nucleotide polymorphisms (SNPs) in 24 HLA class III genes between HLA-E and TAPBP. Gene haplotype blocks were created from individual SNPs using the statistical software PHASE. ${ }^{24}$ There were significant mismatches at several sites. These included single haplotype mismatches at MICB, TNF, HSP70, NOTCH4, and LMP2 and a double haplotype mismatch at LMP7. However, the donor and recipient did not carry the 8.1 HLA haplotype previously associated with colonic CD. ${ }^{2}$ We also genotyped the donor and recipient for three SNPs (IGR2060a_l C/G, IGR2198a_l G/C, IGR3096a_l C/T) spanning the $\overline{\mathrm{IBD}} 5$ susceptibility locus, which encompasses a number of cytokine genes involved in Th1/Th2 regulation. ${ }^{25}$ The CD associated haplotype was not identified in either the donor or pre ASCT recipient.

In summary, although the IBD3 or IBD5 haplotypes associated with CD were not carried by the ASCT donor, significant HLA class III gene mismatches at IBD3 and a CD associated polymorphism of the 5' UTR of NOD2/CARD15 were detected in the donor and in the reconstituted immune cell population of the recipient post ASCT. These genetic data cannot prove that adoptive transfer of $\mathrm{CD}$ susceptibility occurred in this case but they are at least compatible with this phenomenon. Of crucial importance for the hypothesis that donor-recipient transfer of CD susceptibility occurred is the fact that the donor denied any personal or family history of IBD. Therefore, one would have to postulate that development of CD could not have been caused by transfer of donor CD susceptibility genes alone, in this case, but would require an additional factor(s) such as a recipient non-haematopoietic (possibly epithelial cell) CD susceptibility gene polymorphism (which is compatible with CD being a polygenic disease) or an altered environmental factor present for the recipient but not donor (for example, diet or intestinal flora).

As well as stimulating debate about whether adoptive transfer of $\mathrm{CD}$ can occur, this case report also raises questions about current policy regarding ASCT donor selection. Currently there is no donor screening for a personal or family history of IBD. We suggest that formal questioning about IBD should be included during ASCT donor ascertainment, along with careful follow up of previous recipients of an ASCT, from a donor with a positive personal or family history of IBD, in order to investigate further a possible link between ASCT and development of CD. 


\section{Authors' affiliations}

S A Sonwalkar*, M A Hull, Molecular Medicine Unit, University of Leeds, and Department of Gastroenterology, St James's University Hospital, Leeds, UK

R M James*, D L Barnard, Department of Haematology, St James's University Hospital, Leeds, UK

T Ahmad, L Zhang, D P Jewell, Gastroenterology Unit, University of Oxford, Radcliffe Infirmary, Oxford, UK

C S Verbeke, Department of Histopathology, St James's University Hospital, Leeds, UK

*S A Sonwalkar and R M James contributed equally to this work.

Correspondence to: M A Hull, Molecular Medicine Unit, Clinical Sciences Building, St James's University Hospital, Leeds LS9 7TF, UK; M.A.Hull@leeds.ac.uk

Accepted for publication 30 May 2003

\section{REFERENCES}

1 Katz JA, Fiocchi C. Causes and mechanisms of Crohn's disease. In: Prantera C, Korelitz BI, eds. Crohn's disease. New York: Dekker, 1996:9-56.

2 Ahmad T, Armuzzi A, Bunce M et al. The molecular classification of the clinical manifestations of Crohn's disease. Gastroenterology 2002; 122:854-66.

3 Lopez-Cubero SA, Sullivan KM, Mcdonald GB. Course of Crohn's disease after allogenic bone marrow transplantation. Gastroenterology 1998:114:433-40.

4 Soderholm JD, Malm C, Juliusson G, et al. Long-term endoscopic remission of Crohn's disease after autologous stem cell transplantation for acute myeloid leukaemia. Scand J Gastroenterol 2002;37:613-16.

5 Grieco MB, Bordan DL, Geiss AC, et al. Toxic megacolon complicating Crohn's colitis. Ann Surg 1980;191:75-80.

6 lqbal N, Salzman D, Lazenby AJ, et al. Diagnosis of gastrointestinal graftversus-host disease. Am J Gastroenterol 2000;95:3034-8

7 Asplund S, Gramlich TL. Chronic mucosal changes of the colon in graft-versushost disease. Mod Pathol 1998:11:513-15.

8 Naik HR, Chandrasekar PH. Herpes simplex virus (HSV) colitis in a bone marrow transplant recipient. Bone Marrow Transplant 1996;17:285-6.

9 Baden LR, Maguire JH. Gastrointestinal infections in the immunocompromised host. Infect Dis Clin North Am 2001;15:639-70.
10 Bavaro MF. Neutropenic enterocolitis. Curr Gastroenterol Rep 2002; 4:297-301.

11 Aldouri MA, Ruggier R, Epstein O, et al. Adoptive transfer of hyperthyroidism and autoimmune thyroiditis following allogeneic bone marrow transplantation for chronic myeloid leukaemia. Br J Haematol 1990;74:118-19.

12 Neumeister P, Strunk D, Afelbeck U, et al. Adoptive transfer of vitiligo after allogeneic bone marrow transplantation for non-Hodgkin's lymphoma. Lance 2000;355: 1334-5.

13 Snowden JA, Heaton DC. Development of psoariasis after syngeneic bone marrow transplant from psoriatic donor: further evidence for adoptive autoimmunity. Br J Dermatol 1997;137:130-2.

14 Spiers AS. Ulcerative colitis after bone-marrow transplantation for acute leukaemia. N Engl J Med 1259.

15 Baron FA, Hermanne JP, Dowlatti A, et al. Bronchiolitis obliterans organizing pneumonia and ulcerative colitis after allogeneic bone marrow transplantation. Bone Marrow Transplant 1998:21:951-4.

16 Spiers AS. Bone marrow transplantation, ulcerative colitis and acquired immunodeficiency syndrome (AIDS). JAMA 1987;257:1902.

17 James SP. Allogenic bone marrow transplantation in Crohn's disease. Gastoenterology 1998;114:596-8.

18 Breban M, Fernando-Sueiro JL, Richardson JA, et al. T cells, but not thymic exposure to HLA-B27, are required for the inflammatory disease of HLA-B27 transgenic rats. J Immunol 1996; 156:794-803.

19 Simpson SJ, Mizoguchi E, Allen D, et al. Evidence that $C D 4^{+}$, but not $C D 8^{+}, T$ cells are responsible for murine interleukin-2-defecient colitis. Eur J Immunol 1995;25:2618-25.

20 Aranda R, Sydora BC, McAllister PL, et al. Analysis of intestinal lymphocytes in mouse colitis mediated by transfer of CD4 $4^{+}, \mathrm{CD} 45 \mathrm{RB}$ high T cells to SCID recipients. J Immunol 1997; 158:3464-73.

21 Lesage S, Zouali H, Cezard JP, et al. CARD15/NOD2 mutational analysis and genotype-phenotype correlation in 612 patients with inflammatory bowel disease. Am J Hum Genet 2002:70:845-57.

22 Cuthbert AP, Fisher SA, Mirza MM, et al. The contribution of NOD2 gene mutations to the risk and site of disease in inflammatory bowel disease. Gastroenterology 2002;122:867-74.

23 Forcione DG Sands B, Isselbacher KJ, et al. An increased risk of Crohn's disease in individuals who inherit the HLA class II DRB3*0301 allele. Proc Nat Acad Sci U S A 1996;93:5094-8.

24 Stephens M, Smith NJ, Donnelly P. A new statistical method for haplotype reconstruction from population data. Am J Hum Genet 2001;68: 978-89.

25 Rioux JD, Daly MJ, Silverberg MS, et al. Genetic variation in the $5 q 31$ cytokine gene cluster confers susceptibility to Crohn disease. Nat Genet $2001 ; 29: 223-8$. 